Bull. Austral. Math. Soc.

VoL. 48 (1993) [257-264]

\title{
ON CO-FPF MODULES
}

\author{
LE VAN ThuYeT
}

\begin{abstract}
$A$ ring $R$ is called right co-FPF if every finitely generated cofaithful right $R$-module is a generator in mod- $R$. This definition can be carried over from rings to modules. We say that a finitely generated projective distinguished right $R$-module $P$ is a coFPF module (quasi-co-FPF module) if every P-finitely generated module, which finitely cogenerates $\mathrm{P}$, generates $\sigma[\mathrm{P}]$ ( $\mathrm{P}$, respectively). We shall prove a result about the relationship between a co-FPF module and its endomorphism ring, and apply it to study some co-FPF rings.
\end{abstract}

\section{INTRODUCTION}

In this note all rings are associative with identities and all modules are unitary. Let $M_{R}$ be a right $R$-module. A module $N$ is called $M$-generated or $M$ generates $N$ if there exist a set $A$ and an epimorphism $M^{(A)} \rightarrow N$, where $M^{(A)}$ is the direct sum of $|A|$ copies of $M(|A|$ denotes the cardinality of the set $A)$. When $A$ is finite, we say that $N$ is $M$-finitely generated. $N$ is called $M$-cogenerated or $M$ cogenerates $N$ if there exist a set $A$ and a monomorphism $N \rightarrow M^{A}$, where $M^{A}$ is the direct product of $|A|$ copies of $M$. When $A$ is finite, we say that $N$ is $M$-finitely cogenerated. Let $M_{R}$ and $U_{R}$ be two modules. Then $M$ is called $U_{R}$-distinguished if for every nonzero homomorphism $h: X_{R} \rightarrow M$ from a module $X_{R}$ into $M$ there exists a homomorphism $g: U \rightarrow X$ so that $h g \neq 0$. A module $M_{R}$ is distinguished if $M$ is $M$-distinguished. For a module $M_{R}$, we denote by $\sigma[M]$ the full subcategory of $\bmod -R$ whose objects are submodules of $M$-generated modules (see [11]).

For a right $R$-module $M$, the trace ideal of $M$ in $R$ is denoted by trace $(M)$. By definition, $\operatorname{trace}(M)=\sum\left\{\operatorname{im} \varphi, \varphi \in \operatorname{Hom}_{R}\left(M, R_{R}\right)\right\}$ (see $[11$, p.154]).

A module $M_{R}$ is called faithful if $\{a \in R ; M a=0\}=0$. Then $M$ is faithful if and only if $M$ cogenerates every projective right $R$-module. Dually, a module $M_{R}$ is called cofaithful if $M$ generates every injective right $R$-module. It follows that $M$ is cofaithful if and only if there exists a finite subset $\left\{m_{1}, \ldots, m_{n}\right\}$ of elements of $M$ such that $\left\{x \in R, m_{1} x=\cdots=m_{n} x=0\right\}=0$.

Received 22nd September 1992

I wish to thank my supervisor, Professor Dinh van Huynh, for many helpful and stimulating discussions during the preperation of this paper.

Copyright Clearance Centre, Inc. Serial-fee code: 0004-9729/93 \$A2.00+0.00. 
A ring $R$ is called right FPF if every finitely generated faithful right $R$-module is a generator in mod- $R$. FPF rings have been the subject of much research and two recent monographs, Faith and Page [3] and Faith and Pillay [4], have been devoted to them. We introduce the family of right co-FPF rings as a generalisation of the class of right self-injective rings and the class of right FPF rings: A ring $R$ is called right co-FPF if every finitely generated cofaithful right $R$-module is a generator in mod- $R$. Basic results about co-FPF rings were obtained in [6].

The definition of FPF ring was carried over from rings to modules by Page [8] as follows: A finitely generated projective distinguished $R$-module $P_{R}$ is called FPF if every $P$-finitely generated module, which cogenerates $P$, generates $P$. Also we say that a finitely generated projective distinguished $R$-module $P_{R}$ is a (quasi-) co-FPF module if every $P$-finitely generated module, which finitely cogenerates $P$, generates $\sigma[P](P$, respectively). Thus an FPF module is a quasi-co-FPF module, but the converse is not true in general (see [6, Example 2.3]). Since a self-generator is distinguished, a ring $R$ is right co-FPF if and only if $R_{R}$ is a quasi-co-FPF module if and only if $R_{R}$ is a co-FPF module.

Page $[8$, Theorem 4] proved a result about the relationship between a FPF module and its endomorphism ring. Motivated by this, we show in this paper that if $P$ is a finitely generated distinguished projective right $R$-module, then:

(i) If $P$ is a quasi-co-FPF module, then $\operatorname{End}_{R}(P)$ is a right co-FPF ring;

(ii) If $P$ is a self-generator and $\operatorname{End}_{R}(P)$ is a right co-FPF ring, then $P$ is a co-FPF module.

From this it follows that if $R$ is a right co-FPF ring and $e$ a semicentral idempotent of $R$ (that is, $e R=e R e$ ), then $e R=e R e$ is a right co-FPF ring.

\section{RESULTS}

First we list some known results used in this section.

Lemma 1. Let $P_{R}$ be a finitely generated projective right $R$-module with $S=\operatorname{End}\left(P_{R}\right)$. Then:

(i) ${ }_{S} P_{R}$ is an $(S, R)$-bimodule, ${ }_{R} P_{S}^{*}=\operatorname{Hom}_{R}\left(P_{R}, R\right)$ is an $(R, S)$-bimodule, (ii) $F=-\bigotimes_{R} P^{*}$ is a functor from right $R$-modules to right $S$-modules, $G=-\otimes_{S} P$ is a functor from right $S$-modules to right $R$-modules, $H=$ Hom $_{S}\left(P^{*},-\right)$ is a functor from right $S$-modules to right $R$-modules. The functors $G, F, H$ form an adjoint triple $(G, F, H)$ and there are natural transformations $\alpha: 1_{S} \longrightarrow F G, \alpha^{\prime}: F H \longrightarrow 1_{S}, \beta: 1_{R} \longrightarrow H F$, and $\beta^{\prime}: G F \longrightarrow 1_{R}$. 
(iii) There are the evalution homomorphisms:

$$
\begin{array}{r}
\nu: P^{*} \otimes S P \\
f \otimes P \\
\theta: P \otimes_{R} P^{*} \longrightarrow S \\
p \otimes f \longmapsto p f()
\end{array}
$$

and $\theta$ is an isomorphism.

(iv) Set $T=\operatorname{trace}(P)$, then $T=\operatorname{trace}\left(P^{*}\right), P T=P, T P^{*}=P^{*}$ and $P^{*}$ is a finitely generated projective left $R$-module.

(v) $\nu$ is an epimorphism if and only if $P_{R}$ is a generator.

(vi) $P_{S}$ is always a generator over $S$ and $P$ is finitely generated over $S$ if and only if $P$ finitely cogenerates $R$, that is, $P$ is a cofaithful right $R$-module.

Proof: (i), (ii), (iv) and (v) can be proved easily. The statements (iii) and (vi) are proved in $[1,11.19 .1]$ and $[2,19.14 \mathrm{~B}]$, respectively.

Lemma 2. (Kato [5, Lemma 3]) Let $M_{R}$ and $U_{R}$ be two right $R$-modules. Then $M_{R}$ is $U$-distinguished if and only if for each $m \in M, m$.trace $(U)=0$ implies $m=0$.

Recall that a submodule $M^{\prime}$ of $M$ is a pure submodule if the exact sequence

$$
0 \rightarrow M^{\prime} \rightarrow M \rightarrow M / M^{\prime} \rightarrow 0
$$

is pure, that is, $M / M^{\prime}$ is flat.

Lemma 3. (Zimmermann - Huisgen [10, Theorem 2.4]) Let $P_{R}$ be a right $R$ module such that $P$.trace $(P)=P$. Then trace $(P)$ is left pure if and only if $P_{R}$ is a self generator.

Lemma 4. (Rutter [9]) Let $P_{R}$ be a finitely generated projective module and $M$ be a right $R$-module. Then $M \otimes_{R} P^{*}=0$ if and only if $M$.trace $(P)=0$. Moreover, if $M$ is injective and $P$ is distinguished, then $\left(M \otimes_{R} P^{*}\right)_{S}$ is injective .

Lemma 5. (Miller [7, Corollary 2.6 and Theorem 2.7]) Let $P_{R}$ be a finitely generated projective module. If trace $(P)$ is left flat, then $P^{*} \otimes_{S} P \simeq \operatorname{trace}(P)$ as an $(R, R)$-module.

Lemma 6. (Thuyet [6, Lemma 2.1]) Let $M_{R} \in \bmod -R$. Then the following conditions are equivalent:

(i) $M_{R}$ is cofaithful;

(ii) There exists a finite set $\left\{m_{1}, \ldots, m_{n}\right\}$ of elements of $M$ such that $\left\{x \in R, m_{1} x=\cdots=m_{n} x=0\right\}=0$; 
(iii) There exists a positive integer $n$ such that $R_{R}$ can be embedded into $M^{n}$;

(iv) $M$ generates every injective right $R$-module;

(v) $\sigma[M]=\bmod -R$;

(vi) Cyclic submodules of $M^{(\mathrm{N})}$ form a set of generators in mod-R.

Now we state a result about the relationship between a co-FPF module and its endomorphism ring.

THEOREM 7. Let $P$ be a finitely generated distinguished projective right $R$ module. Then :

(i) If $P$ is a quasi-co-FPF module, then $S=\operatorname{End}_{R}(P)$ is a right co-FPF ring.

(ii) If $P$ is a self-generator and $S=\operatorname{End}_{R}(P)$ is a right co-FPF ring, then $P$ is a co-FPF module.

Proof: (i) Assume that $P_{R}$ is quasi-co-FPF and $S=\operatorname{End}_{R}(P)$. Let $M$ be a finitely generated cofaithful right $S$-module. Then we have an exact sequence in mod- $S$ :

$$
S^{n} \longrightarrow M \longrightarrow 0
$$

for some postive integer $n$. Tensoring with ${ }_{S} P_{R}$ gives an exact sequence

$$
\left(S^{n} \otimes_{S} P\right)_{R} \rightarrow\left(M \otimes_{S} P\right)_{R} \rightarrow 0 .
$$

But it is clear that $P_{R}^{n} \simeq\left(S \otimes_{S} P\right)^{n} \simeq\left(S^{n} \otimes_{S} P\right)_{R}$. This proves that $P_{R}$ finitely generates $M \otimes_{S} P$.

Since $M_{S}$ is cofaithful, we have an exact sequence in $\bmod -S$ :

$$
0 \longrightarrow S \stackrel{g}{\longrightarrow} M^{l}
$$

for some positive integer $l$, and the homomorphism

$$
P_{R} \simeq\left(S \otimes_{S} P\right)_{R} \stackrel{f=g \otimes i d}{\longrightarrow} M^{l} \otimes_{S} P
$$

induces an exact sequence:

$$
0 \longrightarrow \operatorname{ker} f \longrightarrow P \stackrel{f}{\longrightarrow} M^{l} \otimes_{S} P .
$$

Now by Lemma 1(iv), $P^{*}$ is a finitely generated projective left $R$-module, hence ${ }_{R} P^{*}$ is flat. Hence the following sequence is exact:

$$
0 \longrightarrow \operatorname{ker} f \otimes_{R} P^{*} \longrightarrow P \otimes_{R} P^{*} \longrightarrow\left(M^{l} \otimes_{S} P\right) \otimes_{R} P^{*} .
$$


By Lemma 1 we have the commutative diagram with exact rows:

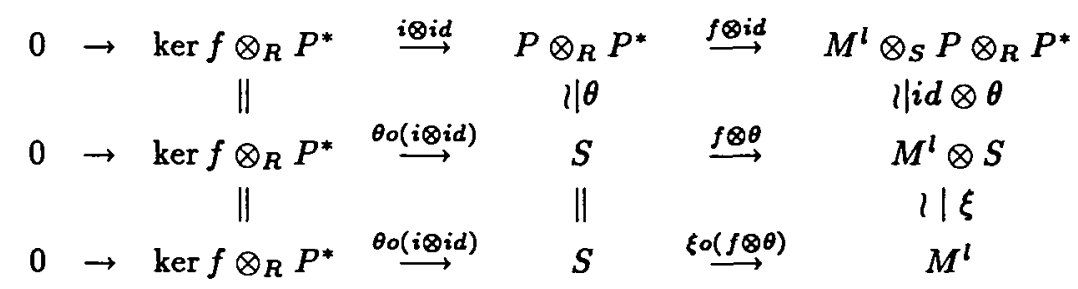

where $\theta$ is defined in Lemma 1 (iii), and $\xi$ is the canonical isomorphism. However $\operatorname{ker}(\xi \circ(f \otimes \theta))=(f \otimes \theta)^{-1} \operatorname{ker} \xi=\operatorname{ker}(f \otimes \theta)=\operatorname{im} f \otimes \operatorname{ker} \theta=0$, hence $0=\operatorname{im}\left(\theta o\left(i \otimes i d_{P^{*}}\right)\right)=\theta(\operatorname{im}(i \otimes i d))=\theta\left(\operatorname{ker} f \otimes P^{*}\right)$. It follows that $\operatorname{ker} f \otimes P^{*}=0$. Let $T=\operatorname{trace}(P)$. Then by Lemma 4 , $\operatorname{ker} f . T=0$ and since $P$ is distinguished, by Lemma 2 , it follows that $\operatorname{ker} f=0$, that is, we have an exact sequence:

$$
0 \longrightarrow P_{R} \longrightarrow\left(M^{l} \otimes_{S} P\right)_{R} \simeq\left(M \otimes_{S} P\right)_{R}^{l} .
$$

Hence $\left(M \otimes_{S} P\right)_{R}$ finitely cogenerates $P$. By assumption, $M \otimes_{S} P$ generates $P$, but since $P$ is finitely generated, $M \otimes s P$ finitely generates $P$, that is, there exists a positive integer $h$ such that

$$
\left(M \otimes_{S} P\right)^{h} \longrightarrow P \longrightarrow 0
$$

is exact in $\bmod -R$. This gives

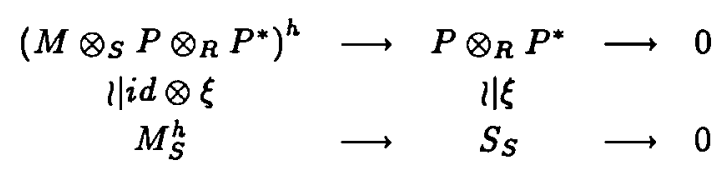

in which the rows are exact. This shows that $M$ generates $S$. Thus $S$ is a right co-FPF ring.

(ii) Assume that $P$ is a self-generator and $S=\operatorname{End}_{R}(P)$ is a right co-FPF ring. We note that $P_{R}$ is a self-generator if and only if trace $P=T_{R}$ is pure in ${ }_{R} R$ if and only if $R(R / T)$ is flat (see [10, Theorem 2.4]). To prove that $P_{R}$ is quasi-co-FPF, let $N_{R}$ be a $P$-finitely generated right $R$-module and $N$ finitely cogenerate $P$. Then we have two exact sequences:

$$
\begin{gathered}
P^{m} \longrightarrow N \longrightarrow 0, \quad m \in \mathbb{N} \\
0 \longrightarrow P \longrightarrow N^{l}, \quad l \in \mathbb{N} .
\end{gathered}
$$

Put $V_{S}=N \otimes_{R} P_{S}^{*}$. The proof of (ii) is divided into four steps. 
STEP 1. $V_{S}$ is cofaithful. In fact, since ${ }_{R} P^{*}$ is flat, and from (2), we obtain:

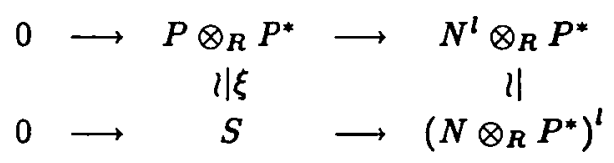

with exact rows. This gives that $V_{S}$ is cofaithful.

STEP 2. $V$ is $S$-finitely generated. In fact, from (1), we obtain the following commutative diagram with exact rows:

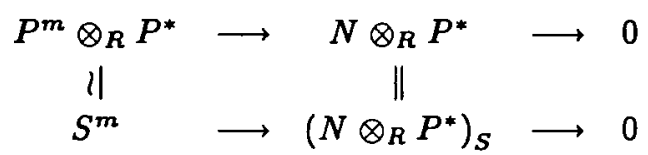

that is, $V$ is $S$-finitely generated.

STEP 3. $N$ finitely generates $P$. From steps 1 and 2 and by assumption, $N \otimes_{R} P^{*}$ is a generator in mod- $S$, that is, there exists an exact sequence in mod- $S$ :

$$
\left(N \otimes_{R} P^{*}\right)^{n} \longrightarrow S \longrightarrow 0
$$

and this yields

$$
\begin{aligned}
& \left(N \otimes_{R} P^{*}\right)^{n} \otimes_{R} P_{R} \longrightarrow S \otimes_{S} P \longrightarrow 0 \\
& \| k \quad \text { l| } \\
& \left(N \otimes_{R} T\right)^{n} \quad \longrightarrow \quad P_{R} \quad \longrightarrow 0
\end{aligned}
$$

where the existence of isomorphism $k$ is obtained from Lemma 5. From this to show that $N$ generates $P$ it is enough to show that $N \simeq N \otimes_{R} T$. We consider the exact sequence:

$$
0 \longrightarrow T \longrightarrow R \longrightarrow R / T \longrightarrow 0
$$

Since $T$ is left pure,

$$
0 \longrightarrow N \otimes T \longrightarrow N \otimes R \longrightarrow N \otimes(R / T) \longrightarrow 0
$$

is exact.

Note that $P T=T$. Thus $0=(P \otimes R / T) T=P T \otimes R / T=R \otimes R / T . \mathrm{By}(1)$, we obtain the commutative diagram with exact rows:

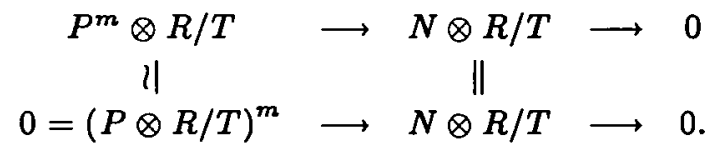

Thus $N \otimes R / T=0$. From this and (3), we obtain the following exact sequences:

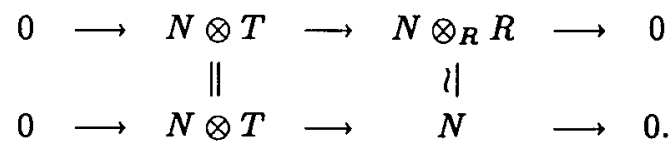

Hence $N \simeq N \otimes_{R} T$. 
STEP 4. By $[11,18.5], P$ is a generator of $\sigma[P]$. Hence by step $3, N$ is also a generator in $\sigma[P]$. This proves that $P$ is a co-FPF module.

Now we have some applications to co-FPF rings.

Proposition 8. Let $R$ be a right co-FPF ring and $e$ an idempotent of $R$ such that $e R$ is distinguished. Then $e R$ is a quasi-co-FPF module and $e R e$ is a right co-FPF ring.

ProOF: Let $M$ be a $e R$-finitely generated module such that it finitely cogenerates $e R$. Hence we have two exact sequences,

$$
\begin{aligned}
& (e R)^{n} \stackrel{f}{\longrightarrow} M \longrightarrow 0 \\
& 0 \longrightarrow e R \stackrel{g}{\longrightarrow} M^{l} .
\end{aligned}
$$

From (1) we obtain another exact sequence

$$
R^{n} \longrightarrow M \longrightarrow 0 \text {. }
$$

It follows that $M$ is $R$-finitely generated. Now we set $U=M \oplus(1-e) R$, then $U$ is also $R$-finitely generated. And from (2) we construct a homomorphism $k$ as follows:

$$
k: R \longrightarrow M^{l} \oplus(1-e) R
$$

with $k=g \oplus i d_{(1-e) R}$. It is easy to see that $k$ is a monomorphism. From this and the inclusion map $j: M^{l} \oplus(1-e) R \hookrightarrow(M \oplus(1-e) R)^{l}$ we obtain that $j k$ is a monomorphism from $R_{R}$ to $(M \oplus(1-e) R)^{l}$. This shows that $M \oplus(1-e) R$ is a cofaithful module. By assumption $U=M \oplus(1-e) R$ is a generator for mod- $R$, in particular $U$ generates $e R$. Thus

$$
\operatorname{trace}_{e R}(M \oplus(1-e) R)=\operatorname{trace}_{e R} M+\operatorname{trace}_{e R}(1-e) R=\operatorname{trace}_{e R} M=e R,
$$

that is, $M$ generates $e R$ or equivalently, $e R$ is a quasi-co-FPF module. This together with Theorem 7 shows that $\operatorname{End}_{R}(e R) \simeq e R e$ is right co-FPF.

Lemma 9. If $e$ is a right semicentral idempotent, then $e R$ is distinguished.

Proof: For every er $\in e R$, if er.trace $(e R)=0$ then $e r R e R=0$ and hence $e r e=0$. Since $e$ is a right semicentral idempotent, er $=e r e=0$. By Lemma $2, e R$ is distinguished.

Corollary 10. Let $R$ be a right co-FPF ring, and e be a right semicentral idempotent. Then $e$ Re is a right co-FPF ring.

Proof: By Proposition 8 and Lemma 9.

Corollary 11. If $e$ is an idempotent of $R$ such that $e R$ is distinguished, selfgenerator and $e R e$ is a right co-FPF ring, then $e R$ is a co-FPF module.

Proof: By Theorem 7 . 


\section{REFERENCES}

[1] C. Faith, Algebra I : Rings, modules and categories. (Springer-Verlag, Berlin, Heidelberg, New York, 1973).

[2] C. Faith, Algebra II : Ring theory (Springer-Verlag, Berlin, Heidelberg, New York, 1976).

[3] C. Faith and S. Page, FPF ring theory: Faithful Modules and generators of Mod-R, London Math. Soc. Lecture Notes 88 (Cambridge Univ. Press, Cambridge, 1984).

[4] C. Faith and P. Pillay, Classification of commutative FPF rings, Notas de Matematica 4 (Universidad de Murcia, 1990).

[5] T. Kato, 'U-distinguished modules', J. Algebra 25 (1973), 15 - 24.

[6] L.V. Thuyet, 'On rings whose finitely generated cofaithful modules are generators', Algebra Ber. (1993) (to appear).

[7] R.W. Miller, 'Finitely generated projective modules and TF classes', Pacific. J. Math. 64 (1976), 505-515.

[8] S. Page, 'FPF endomorphism rings with applications to QF-3 rings', Comm. Algebra 14 (1986), 423-435.

[9] E.A. Rutter Jr., 'PF-modules', Tôhoku Math. J. 23 (1971), 201 - 206.

[10] B. Zimmermann-Huisgen, 'Endomorphism rings of self-generators', Pacific J. Math. 61 (1975), 587 - 602 .

[11] R. Wisbauer, Grundlagen der Modul- und Ringtheorie (R. Fischer Verlag, München, 1988).

Department of Mathematics

Hue Teachers' Training College

32 Le Loi St

Hue

Vietnam 